\title{
Severe pulmonary mineralization in a dog with pituitary-dependent hyperadrenocorticism: a case report
}

\author{
Ji-Seon Yoon ${ }^{1}$, Seongwoo Kang ${ }^{2}$, Jinho Park ${ }^{1}$, Jihye Choi ${ }^{3, *}$, DoHyeon Yu ${ }^{2, *}$ \\ ${ }^{l}$ Department of Veterinary Internal Medicine, College of Veterinary Medicine, Chonbuk National University, Iksan 54596, Korea \\ ${ }^{2}$ Department of Veterinary Internal Medicine, College of Veterinary Medicine, Gyeongsang National University, \\ Jinju 52828, Korea \\ ${ }^{3}$ Department of Veterinary Diagnostic Imaging, College of Veterinary Medicine, Chonnam National University, \\ Gwangju 61186, Korea
}

\begin{abstract}
A 7-year-old, spayed female Chihuahua with dyspnea, intermittent cyanosis, and dermal plaques was diagnosed with hyperadrenocorticism. Thoracic radiographs showed markedly hyperdense alveoli in multiple lung lobes. Computed tomography (CT) images showed lung mineralization confined to the lung parenchyma and pituitary macroadenoma. Pulmonary mineralization secondary to hyperadrenocorticism is commonly found on histopathologic examination. However, those lesions are rarely identified radiographically. We describe obvious pulmonary mineralization secondary to hyperadrenocorticism found on radiographs and present the first report regarding CT imaging of the mineralization. Pulmonary mineralization should be considered when a dog affected by hyperadrenocorticism shows diffuse lung mineralization on radiographs.
\end{abstract}

Keywords: computed tomography, dog, hyperadrenocorticism, mineralization, radiography

\section{*Corresponding author}

DoHyeon Yu

College of Veterinary Medicine, Gyeongsang National University, 501 Jinju-daero, Jinju 52828 , Korea

Tel.: $+82-55-772-2368$

Fax: $+82-55-772-2330$

E-mail: yudh@gnu.ac.kr

Jihye Choi

College of Veterinary Medicine, Chonnam National University, 77 Yongbong-ro, Buk-gu, Gwangju 61186, Korea

Tel.: $+82-62-530-2821$

Fax: +82-62-530-2881

E-mail: imsono@jnu.ac.kr

ORCID:

Jiseon Yoon

https://orcid.org/0000-0002-7354-3255

Seongwoo Kang

https://orcid.org/0000-0001-9893-9275

Jinho Park

https://orcid.org/0000-0001-5235-5717

Jihye Choi

https://orcid.org/0000-0002-1258-7158

Do Hyeon $\mathrm{Yu}$

https://orcid.org/0000-0001-7645-6926

Conflict of Interest

The authors declare no conflicts of interest.

Received: May 25, 2019

Revised: July 29, 2019

Accepted: October 29, 2019
Pulmonary mineralization is a common pathologic finding in dogs with hyperadrenocorticism [1]. However, antemortem diagnosis of pulmonary mineralization is extremely difficult, as it generally does not induce remarkable change on radiography. Calcification of the tracheal rings and bronchial walls can be observed radiographically in dogs with hyperadrenocorticism, but these can also be age-related changes [2,3]. Compared with airway calcification, pulmonary mineralization is usually seen as a generalized interstitial lung pattern, not as diffuse mineral densities.

Due to the low sensitivity of radiography for pulmonary mineralization, 3 dogs with hyperadrenocorticism have previously been diagnosed using nuclear scintigraphy with 99m-technitium-methylene diphosphonate $[4,5]$. In human medicine, not only is nuclear scintigraphy used, but high-resolution computed tomography (CT) is also recommended for the definitive diagnosis of pulmonary mineralization [2,6]. Pulmonary mineralization may contribute to hypoxemia in dogs [4], thus CT study should be considered in any dog with suspected pulmonary mineralization from hyperadrenocorticism. Though CT imaging is widely available in veterinary medicine, to the authors' knowledge, there are no reports on $\mathrm{CT}$ imaging of pulmonary mineralization in dogs with hyperadrenocorticism. Here, we describe a case of pulmonary mineralization secondary to pituitary-dependent hyperadrenocorticism (PDH) as visualised by radiography and $\mathrm{CT}$ study.

A 7-year-old, spayed female Chihuahua presented in respiratory distress with cutaneous lesions. Erythematous, palpable, and firm papules as well as welldemarcated plaques with ulceration and crusting were observed on the thorax and abdominal region (Fig. 1A). Respiratory signs including dyspnea, panting and cyanosis under stress had been observed for months. Previous diagnostic examination or treatment had not been pursued in this dog because the clinical signs were mild. A complete blood count showed mild thrombocytosis $(614 \mathrm{~K} / \mu \mathrm{L}$; normal range, $148-484 \mathrm{~K} / \mu \mathrm{L})$ with a stress leukogram demonstrating neutrophilia and eosinopenia. Serum chemistry showed a marked elevation of alkaline phosphatase (1,597 U/L; normal range, 23-212 U/L). 


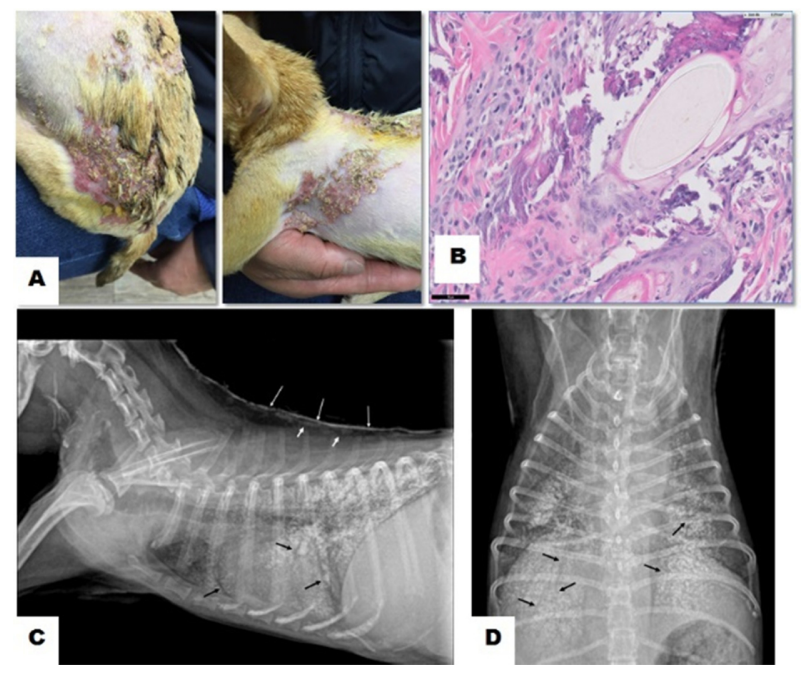

Fig. 1. Clinical, histopathological, and radiographic findings of the present case. Palpable and firm plaques covered with crusts were noted on the skin of the dorsum (A). Basophilic and irregularly shaped mineralized debris was noted in the dermis by histopathological analysis (B). Bar indicates $50 \mu \mathrm{m}$ (hematoxylin and eosin staining). On the lateral (C) and ventrodorsal (D) radiographic views, diffuse mineral density was observed from the overall lung fields particularly from the caudal lung lobes. Air-filled bronchi (black arrows) are clearly differentiated from the radiopaque pulmonary parenchyma. Note the generalized mineralization of the cutaneous (white long arrows) and subcutaneous tissues (white short arrows).

Radiography revealed mineralization with alveolar infiltrate and radiolucent bronchus within the lung lobes, particularly in the caudal lobes, as well as mineralization of tracheal rings (Fig. 1C and D). Dorsal cutaneous and subcutaneous radiopaque changes were also present, suggesting mineralization. Abdominal ultrasonography revealed bilateral mineralization of the renal cortices and bilateral adrenal gland enlargement $($ left $=6.7 \mathrm{~mm}$, right $=5.7 \mathrm{~mm})$. Hepatomegaly with hyperechoic change and fine texture was noted.

CT was performed to evaluate the pulmonary lesions in detail. Due to respiratory distress, the CT study was completed in sternal recumbency without sedation or anaesthesia, using a 16-row, multi-detector CT scanner (Siemens Emotion 16; Siemens, Germany) with the following settings: slice thickness $=1 \mathrm{~mm}$; pitch $=0.8$; rotation duration $=600$ $\mathrm{ms}$; tube voltage $=120 \mathrm{kV}$; and tube current $=120 \mathrm{~mA}$. Contrast enhanced CT images were acquired after intravenous injection of $2 \mathrm{mg} / \mathrm{kg}$ iohexol (Omnipaque 300; GE Healthcare, Norway). In the lung window, (window range 1,200 Hounsfield unit [HU] and window level $-600 \mathrm{HU})$, the overall lung density increased diffusely at about $-370 \mathrm{HU}$, with multifocal hyperattenuation of the pulmonary alveoli. The hyperattenuating pulmonary alveoli were coalesced and had bone attenuation of about $658 \mathrm{HU}$ in all lung lobes except the right cranial lobe (Fig. 2A-F). Gas-filled bronchi were clearly differentiated from the hyperattenuated pulmonary alveoli. The size and shape of pulmonary vessels were within the normal range. In the soft tissue window (window range 400 $\mathrm{HU}$ and window level $40 \mathrm{HU}$ ), there was no enhancement of the lung parenchyma after contrast injection. Extensive mineralization of cutaneous tissues was observed from the cervical to the abdominal region, particularly along the dorsum; mineralized cutaneous tissues were thicker than other regions. Mineralization was confined to the cutaneous layer without expanding into the subcutaneous area. The adrenal glands were enlarged bilaterally and had homogeneous contrast enhancement. There was no evidence of malignant change in the adrenal glands. On intracranial CT images, a mass measuring $11 \times 9 \mathrm{~mm}$ and that was markedly contrast enhanced was found in the region of the pituitary (Fig. $2 \mathrm{G}$ and $\mathrm{H}$ ). The mass was suspected to be a pituitary adenoma. Incidental findings included dilation of the lateral ventricles, third ventricle, and midbrain aqueduct along with a persistent fontanel and small accumulation of fluid within the left tympanic bulla.

To confirm $\mathrm{PDH}$, we performed an adrenocorticotropic hormone $(\mathrm{ACTH})$ stimulation test. The baseline serum cortisol level was $9.3 \mu \mathrm{g} / \mathrm{dL}$ (reference range, $2.0-6.0 \mu \mathrm{g} / \mathrm{dL}$ ), and the post cortisol level was $30 \mu \mathrm{g} / \mathrm{dL}$ (reference range, 6.0 $18.0 \mu \mathrm{g} / \mathrm{dL}$ ) after intravenous administration of $5 \mu \mathrm{g} / \mathrm{kg}$ of tetracosactrin (Synacthen injection; Dalim Biotech, Korea).

Based on the blood test and imaging data, the extensive radiopaque changes of the cutaneous and subcutaneous regions, and lung parenchyma were diagnosed as dystrophic mineralization caused by PDH. The pulmonary mineralization induced the respiratory signs seen in the dog. Punch biopsies of the skin were obtained after local anaesthesia with lidocaine. On microscopic examination, the mineralized debris was deeply basophilic and irregularly shaped (Fig. 1B). The overlying epidermis and follicular epithelium were hyperplastic, and the dermis was densely fibrotic, with some areas of mature granulation tissue. Histopathologic examination was consistent with cutaneous calcinosis cutis.

Hyperadrenocorticism was confirmed and trilostane was administered at a dose of $1 \mathrm{mg} / \mathrm{kg}$ orally every 12 hours. Despite treatment, the respiratory signs persisted. The dog was euthanised due to respiratory distress 60 days after the diagnosis. Unfortunately, necropsy could not be performed due to the owner's wishes.

Pulmonary mineralization is rarely found by radiography as compared with the mineralization of the airway which is usually seen in aging animals. Although about $90 \%$ of hyperadrenocorticism cases have pulmonary mineralization on histopathologic examination, only a few cases have been identified on radiographs $[1,4]$. Even in those dogs, the generalised interstitial lung pattern seen was not specific to pulmonary mineralization. Interstitial infiltrate can be seen in other conditions such as chronic bronchitis, cardiogenic and non-cardiogenic pulmonary oedema, eosinophilic bronchopneumopathy, lung fibrosis and more. In the present case, 


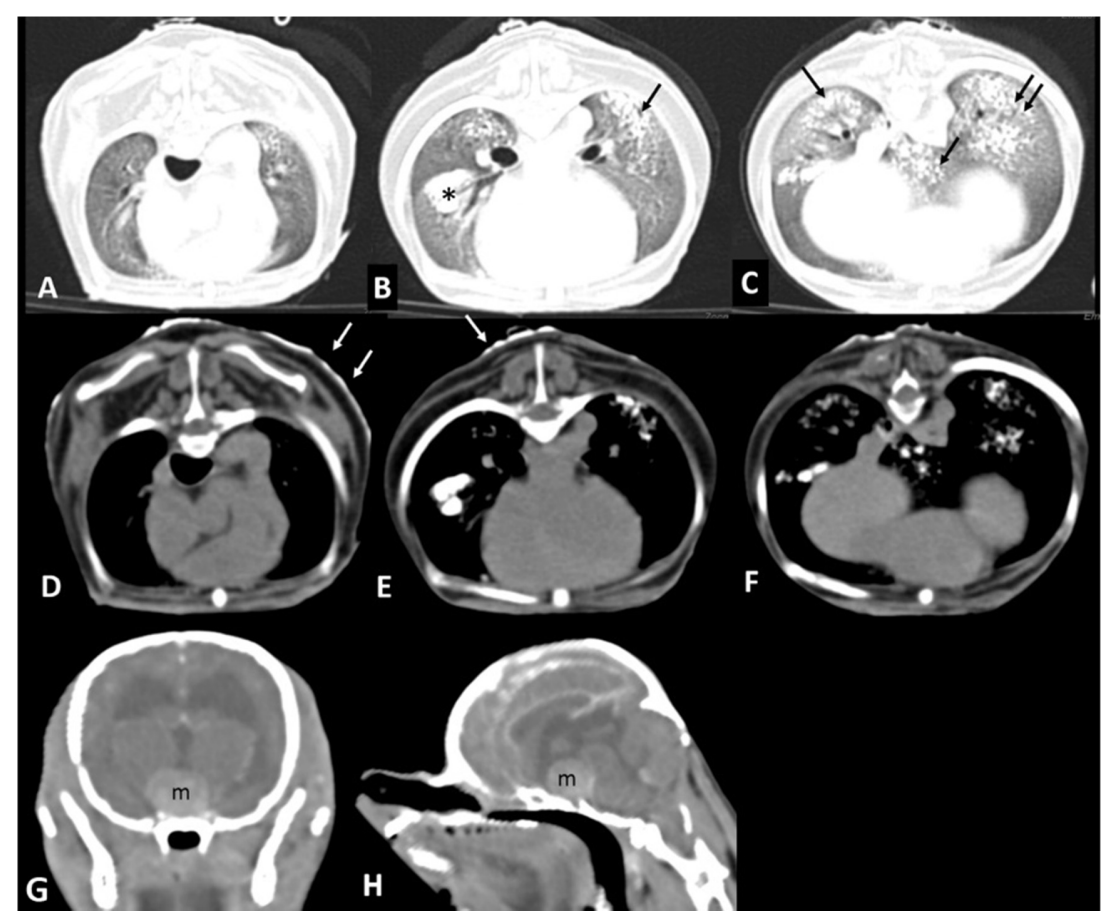

Fig. 2. $\mathrm{CT}$ images of the present case. Transverse plane $\mathrm{CT}$ images (A-F) were obtained. In the lung window (A-C, window range $1,200 \mathrm{HU}$ and window level $-600 \mathrm{HU}$ ), the overall lung fields showed diffuse interstitial infiltrate. In addition, the coalescing mineralized alveoli (black arrows) can be visualised in multifocal areas. In the right middle lobe, the alveoli looked like a mineral mass (asterisk) containing gas-filled bronchus. In the soft tissue window (D-F, window range $400 \mathrm{HU}$ and window level $40 \mathrm{HU}$ ), cutaneous mineralization (white arrows) can be observed along the dorsum. (A-D), (B-E), and (C-F) correspond to the cranial, middle, and caudal lung fields. The left side of the image portrays the right side of the patient. Furthermore, reformatted transverse $(\mathrm{G})$ and sagittal $(\mathrm{H})$ planes of the intracranial CT images were also obtained after contrast injection. In the soft tissue window (window range $400 \mathrm{HU}$, window level $40 \mathrm{HU}$ ), a contrast enhanced mass (m) is observed from the pituitary region. There was no mass effect on the adjacent structures. Hydrocephalus and an open fontanel were found incidentally. CT, computed tomography; HU, Hounsfield unit.

marked hyperdense alveoli were identified in multiple lung lobes and the density of the alveoli was notably high, indicating the possibility of pulmonary mineralization rather than other conditions.

In humans, bone scintigraphy and $\mathrm{CT}$ scans are used to confirm pulmonary mineralization [6,7]. CT allows the identification of minimal changes in lung density as it discriminates a density difference of $0.25 \%$ to $0.5 \%$, as compared to radiography, which can detect a density difference of about $10 \%$ [3]. CT study was performed in our case due to the availability of the imaging modality. In this dog, not only were there mineral density changes of multiple lesions, but also increased density of overall lung fields. In general, lung densities are considered to be normal between -700 and $-850 \mathrm{HU}$ [8]. When hyperinflation or emphysema occurs in the lung, the lung density falls below $-850 \mathrm{HU}$. When the lung density is above $-700 \mathrm{HU}$, infiltrative parenchymal disease is suspected. In this dog, the density of the lung parenchyma was about $-370 \mathrm{HU}$ due to the interstitial infiltrate, which was not observed on radiography. Moreover, discrete lesions with mineral density were observed in multifocal areas on CT images. The lesions were coalesced mineralized alveoli which contained gas-filled bronchi in some areas. The dog in this case presented with dyspnoea and cyanosis under stress and finally died secondary to respiratory distress. In addition to marked mineralized alveoli, diffuse parenchymal infiltrates could have aggravated the respiratory condition in this case.

About $33 \%$ of dogs with pulmonary mineralization showed hypoxemia $\left(\mathrm{PaO}_{2}<53 \mathrm{mmHg}\right)$ in a previous study [4]. In hyperadrenocorticism, respiratory failure, such as panting and dyspnoea, is one of the most common clinical findings. However, thrombosis is another potential complication of the hyperadrenocorticism, with pulmonary thromboembolism (PTE) being most common in dogs [9]. The radiographic features of PTE can be nonspecific ranging from normal appearance to interstitial infiltrate. Chronic thrombi in the pulmonary vessels can be seen as mineral densities, but they are confined to the vascular lumen, not the alveoli. In dogs, clinical signs of pulmonary mineralization are difficult to differentiate from signs of PTE, but the typical CT features, such as increased mineral density of the pulmonary alveoli, can be pathognomonic for pulmonary mineralization.

Lack of the histopathologic examination of the lungs lim- 
its this case. However, the presumptive diagnosis of pulmonary mineralization secondary to $\mathrm{PDH}$ could be made in this case based on the evidence: 1) PDH confirmed by ACTH stimulation testing, adrenal hyperplasia and a pituitary mass, 2) generalised mineralization of the cutaneous tissue confirmed as calcinosis cutis on histopathologic examination, and 3) CT confirmed the mineralization to the pulmonary alveoli and ruled out PTE as the cause of mineral densities in the lung.

We report a dog diagnosed with dystrophic pulmonary mineralization and calcinosis cutis secondary to PDH. To the best of our knowledge, this is the first case of obvious pulmonary mineralization visualised as mineral density on radiography in a dog with PDH, and the first description of CT features associated with pulmonary mineralization. Pulmonary mineralization should be considered when a dog affected by hyperadrenocorticism exhibits respiratory failure and diffuse interstitial infiltrates or mineralization on radiography.

\section{Acknowledgments}

This research was supported by the Basic Science Research Program through the National Research Foundation of Korea (NRF), and funded by the Ministry of Science, ICT \& Future Planning (NRF-2017R1D1A1B03034904).

\section{References}

1. Chan ED, Morales DV, Welsh CH, McDermott MT, Schwarz MI. Calcium deposition with or without bone formation in the lung. Am J Respir Crit Care Med 2002;165:1654-1669.

2. Huntley K, Frazer J, Gibbs C, Gaskell CJ. The radiological features of canine Cushing's syndrome: a review of fortyeight cases. J Small Anim Pract 1982;23:369-380.

3. Marsh S, Aldington S, Williams MV, Nowitz MR, KingzettTaylor A, Weatherall M, Shirtcliffe PM, McNaughton AA, Pritchard A, Beasley R. Utility of lung density measurements in the diagnosis of emphysema. Respir Med 2007;101:15121520.

4. Berry CR, Hawkins EC, Hurley KJ, Monce K. Frequency of pulmonary mineralization and hypoxemia in 21 dogs with pituitary-dependent hyperadrenocorticism. J Vet Intern Med 2000;14:151-156.

5. Blois SL, Caron I, Mitchell C. Diagnosis and outcome of a dog with iatrogenic hyperadrenocorticism and secondary pulmonary mineralization. Can Vet J 2009;50:397-400.

6. Chung MJ, Lee KS, Franquet T, Müller NL, Han J, Kwon OJ. Metabolic lung disease: imaging and histopathologic findings. Eur J Radiol 2005;54:233-245.

7. Belém LC, Zanetti G, Souza AS Jr, Hochhegger B, Guimarães MD, Nobre LF, Rodrigues RS, Marchiori E. Metastatic pulmonary calcification: state-of-the-art review focused on imaging findings. Respir Med 2014;108:668-676.

8. Widmer WR, Guptill L. Imaging techniques for facilitating diagnosis of hyperadrenocorticism in dogs and cats. J Am Vet Med Assoc 1995;206:1857-1864.

9. Kemerink GJ, Kruize HH, Lamers RJ, van Engelshoven JM. Density resolution in quantitative computed tomography of foam and lung. Med Phys 1996;23:1697-1708. 